2014

\title{
Lattice-Boltzmann Simulations of the Thermally Driven 2D Square Cavity at High Rayleigh Numbers
}

\author{
Dario Contrino
}

Pierre Lallemand

Pietro Asinari

Li-Shi Luo

Old Dominion University

Follow this and additional works at: https://digitalcommons.odu.edu/mathstat_fac_pubs

Part of the Applied Mathematics Commons, Computer Sciences Commons, and the Physics Commons

\section{Repository Citation}

Contrino, Dario; Lallemand, Pierre; Asinari, Pietro; and Luo, Li-Shi, "Lattice-Boltzmann Simulations of the Thermally Driven 2D Square Cavity at High Rayleigh Numbers" (2014). Mathematics \& Statistics Faculty Publications. 46.

https://digitalcommons.odu.edu/mathstat_fac_pubs/46

\section{Original Publication Citation}

Contrino, D., Lallemand, P., Asinari, P., \& Luo, L. S. (2014). Lattice-Boltzmann simulations of the thermally driven 2D square cavity at high Rayleigh numbers. Journal of Computational Physics, 275, 257-272. doi:10.1016/j.jcp.2014.06.047 


\title{
Lattice-Boltzmann simulations of the thermally driven 2D square cavity at high Rayleigh numbers
}

\author{
Dario Contrino $^{\mathrm{a}}$, Pierre Lallemand ${ }^{\mathrm{b}}$, Pietro Asinari ${ }^{\mathrm{a}}$, Li-Shi Luo $^{\mathrm{c}, \mathrm{b}}$ \\ ${ }^{a}$ Energy Department, Politecnico di Torino, Torino 10129, Italy \\ ${ }^{b}$ Beijing Computational Science Research Center, Beijing 100084, China \\ ${ }^{c}$ Department of Mathematics 85 Statistics, Old Dominion University, \\ Norfolk, VA 23529, USA
}

\begin{abstract}
The thermal lattice Boltzmann equation (TLBE) with multiple-relaxationtimes (MRT) collision model is used to simulate the steady thermal convective flows in the two-dimensional square cavity with differentially heated vertical walls at high Rayleigh numbers. The MRT-TLBE consists of two sets of distribution functions, i.e., a D2Q9 model for the mass-momentum equations and a D2Q5 model for the temperature equation. The dimensionless flow parameters are the following: the Prandtl number $\mathrm{Pr}=0.71$ and the Rayleigh number $\mathrm{Ra}=10^{6}, 10^{7}$, and $10^{8}$. The D2Q9+D2Q5 MRT-TLBE is shown to be second-order accurate and to be capable of yielding results of benchmark quality, including various Nusselt numbers and local hydrodynamic intensities. The results obtained by using the MRT-TLBE agree well with existing benchmark data obtained by other methods.
\end{abstract}

Key words: Steady Rayleigh-Bénard convective flow, 2D square cavity with differentially heated vertical walls, lattice Boltzmann equation

\section{Introduction}

There has been a systematic effort to construct the lattice Boltzmann equation (LBE) for thermal hydrodynamics since the inception of the lat-

Email address: lluo@odu.edu (Li-Shi Luo) 
tice Boltzmann method (LBM). From early on, it was realized that, while the LBE with energy conservation can lead to the correct macroscopic equations (cf., e.g., [1]), the energy-conserving lattice Boltzmann (LB) models are plagued with severe numerical instability [2], hence they are of little practical use.

It was found that in addition to being prone to numerical instabilities, energy-conserving LBE is also plagued with spurious couplings between energy and shear modes of the linearized evolution operator [2]. To remove this spurious coupling between energy and shear modes, the energy-conservation law must be separated from the mass and momentum conservation laws and there exist at least two approaches to accomplish this goal. The first approach is to use two sets of distribution functions, one for the mass-momentum conservations and the other for the energy conservation (cf., e.g., [3, 4]); and the second is a hybrid approach with the LBE for the mass-momentum conservations and a finite-difference (FD) scheme for the temperature equation $[2,5]$. Both these approaches effectively remove the spurious energy-shear mode coupling. However, the hybrid thermal LBE encounters a new problem - it is difficult to realize the temperature boundary conditions which are consistent with the LB boundary conditions for mass-momentum equations. Consequently, the approach of dual distributions becomes the prevailing one.

In this work we will use the thermal lattice Boltzmann equation (TLBE) with dual distributions and the multiple-relaxation-time collision model to simulate internal thermal flows in two-dimensions (2D). The TLBE consists of a D2Q9 MRT-LBE [6, 7] for the mass-momentum conservations and a D2Q5 MRT-LBE $[8,9,10,11,12]$ for the advection-diffusion equation for the temperature. The TLBE with D2Q9 and D2Q5 LB models has been validated for its accuracy and stability [4]. In particular, we will use the D2Q9+D2Q5 TLBE to simulate the thermally driven convective flow in the $2 \mathrm{D}$ square cavity with differentially heated vertical walls $[13,14]$. This flow has been used to validate various numerical methods including pseudo-spectral (PS) [15], finite difference $(\mathrm{FD})[14,16]$, finite element $(\mathrm{FE})[17,18]$, finite volume (FV) 
$[19,20]$, and control volume (CV) [21] methods. In fact, the 2D square cavity with differentially heated vertical walls has become a standard benchmark problem for incompressible thermal flows. One of the primary purpose of the present work is to reproduce benchmark quality results for the thermally driven steady 2D cavity flow at high Rayleigh numbers $\left(\mathrm{Ra}=10^{6}, 10^{7}\right.$, and $10^{8}$ ) by using the MRT-TLBE model we have developed [4].

The remainder of the paper is organized as follows. Section 2 provides a detailed description of the D2Q9+D2Q5 MRT-TLBE for thermal flows [4], including specifically the details of the MRT-TLBE and the corresponding macroscopic hydrodynamic equations, and the implementation of the TLBE algorithm. Section 3 discusses the flow configuration of the 2D square cavity with differentially heated vertical walls. We pay a special attention to the boundary conditions in the flow and their LB implementations. Section 4 describes the numerical procedures for post-processing, which are used to measure various quantities in the simulations. Section 5 presents our numerical results. We first present a study on the effect of a parameter in the MRT-TLBE which determines the heat diffusivity $\kappa$; we also conduct a convergence study of the flow fields, Nusselt numbers, and some local hydrodynamic intensities; we then compare our results with existing benchmark data. Finally, Section 6 concludes the paper with a summary.

\section{The lattice Boltzmann model for incompressible thermal flows}

The lattice Boltzmann model for incompressible thermal flows consisting of two sets of distributions and their corresponding evolution equations one for conservations of mass and momentum and the other for the temperature - was first proposed by Eggels and Summers [3]. This model assumes Boussinesq approximation, i.e., the temperature $T$ is a passive scalar and its influence to the velocity field $\boldsymbol{u}$ is through the buoyancy term. We will use the LBE with MRT-LBE $[6,7,22,23]$ throughout this work. The MRT-LBE for thermal hydrodynamics has been developed in our previous work [4]. To make the present work self-contained, we will provide the details of the LBE 
model and its implementation in what follows.

\subsection{D2Q9 model for mass and momentum conservations}

The D2Q9 model will be used for the mass and momentum conservations. The discrete velocity set, $\left\{\boldsymbol{c}_{i} \mid i=0,1, \ldots, 8\right\}$, for the D2Q9 model is

$$
\boldsymbol{c}_{i}= \begin{cases}(0,0) & i=0 \\ ( \pm 1,0) c,(0, \pm 1) c, & i=1-4 \\ ( \pm 1, \pm 1) c, & i=5-8\end{cases}
$$

where $c:=\delta_{x} / \delta_{t}$, and $\delta_{x}$ and $\delta_{t}$ are the grid spacing and the time-step size, respectively. The evolution equation is written in general as the following:

$$
\mathbf{f}\left(\boldsymbol{x}_{j}+\boldsymbol{c} \delta_{t}, t_{n}+\delta_{t}\right)=\mathbf{f}\left(\boldsymbol{x}_{j}, t_{n}\right)-\mathbf{M}^{-1} \cdot \mathbf{S} \cdot\left[\mathbf{m}-\mathbf{m}^{(0)}\right]\left(\boldsymbol{x}_{j}, t_{n}\right)+\mathbf{F}\left(\boldsymbol{x}_{j}, t_{n}\right),
$$

where the bold-face font denotes 9-dimensional vectors:

$$
\begin{aligned}
& \mathbf{f}\left(\boldsymbol{x}_{j}+\boldsymbol{c} \delta_{t}, \cdot\right):=\left(f_{0}\left(\boldsymbol{x}_{j}, \cdot\right), f_{1}\left(\boldsymbol{x}_{j}+\boldsymbol{c}_{1} \delta_{t}, \cdot\right), \ldots, f_{8}\left(\boldsymbol{x}_{j}+\boldsymbol{c}_{8} \delta_{t}, \cdot\right)\right)^{\dagger}, \\
& \mathbf{f}\left(\boldsymbol{x}_{j}, \cdot\right):=\left(f_{0}\left(\boldsymbol{x}_{j}, \cdot\right), f_{1}\left(\boldsymbol{x}_{j}, \cdot\right), \ldots, f_{8}\left(\boldsymbol{x}_{j}, \cdot\right)\right)^{\dagger} \\
& \mathbf{m}\left(\boldsymbol{x}_{j}, \cdot\right):=\left(m_{0}\left(\boldsymbol{x}_{j}, \cdot\right), m_{1}\left(\boldsymbol{x}_{j}, \cdot\right), \ldots, m_{8}\left(\boldsymbol{x}_{j}, \cdot\right)\right)^{\dagger}
\end{aligned}
$$

$\mathbf{f}$ and $\mathbf{m}$ are vectors of the discrete-velocity distributions and corresponding moments, respectively, $\mathbf{m}^{(0)}$ is the vector of the equilibrium moments, and $\mathbf{F}$ is the vector of external forcing, which is implemented according to the procedure to be discussed later in Sec. 2.4.

The transformation matrix $\mathrm{M}$ maps the distributions $\left\{f_{i} \mid i=0,1, \ldots, 8\right\}$ to the corresponding moments $\left\{m_{i} \mid i=0,1, \ldots, 8\right\}$, i.e.,

$$
\mathbf{m}=\mathrm{M} \cdot \mathbf{f}, \quad \mathbf{f}=\mathrm{M}^{-1} \cdot \mathbf{m}
$$


Specifically, we use the following $\mathrm{M}$ :

$$
\mathbf{M}=\left(\begin{array}{ccccccccc}
1 & 1 & 1 & 1 & 1 & 1 & 1 & 1 & 1 \\
0 & 1 & 0 & -1 & 0 & 1 & -1 & -1 & 1 \\
0 & 0 & 1 & 0 & -1 & 1 & 1 & -1 & -1 \\
-4 & -1 & -1 & -1 & -1 & 2 & 2 & 2 & 2 \\
0 & 1 & -1 & 1 & -1 & 0 & 0 & 0 & 0 \\
0 & 0 & 0 & 0 & 0 & 1 & -1 & 1 & -1 \\
0 & -2 & 0 & 2 & 0 & 1 & -1 & -1 & 1 \\
0 & 0 & -2 & 0 & 2 & 1 & 1 & -1 & -1 \\
4 & -2 & -2 & -2 & -2 & 1 & 1 & 1 & 1
\end{array}\right)
$$

With $\mathrm{M}$ given above, the ordering of the moments is, as in [4]:

$$
\mathbf{m}=\left(\rho, j_{x}, j_{y}, e, p_{x x}, p_{x y}, q_{x}, q_{y}, \epsilon\right)^{\dagger}
$$

where $\rho$ is the mass density, $\boldsymbol{j}=\rho \boldsymbol{u}$ is the flow momentum and $\boldsymbol{u}$ is the flow velocity; $e$ is the second-order moment corresponding to energy; $p_{x x}$ and $p_{x y}$ are two off-diagonal components of the stress tensor; $q_{x}$ and $q_{y}$ are the third-order moments corresponding to the $x$ and $y$ components of the energy flux, respectively; and $\epsilon$ is the fourth-order moment [6, 7]. The diagonal relaxation matrix is given by:

$$
\mathrm{S}=\operatorname{diag}\left(1,1,1, s_{e}, s_{\nu}, s_{\nu}, s_{q}, s_{q}, s_{\epsilon}\right), \quad s_{i} \in(0,2) \forall i
$$

In what follows we will use the two-relaxation-time (TRT) model due to Ginzburg [8, 24, 25], in which

$$
\begin{aligned}
& s_{e}=s_{\epsilon}=s_{\nu}=\frac{2}{6 \nu+1}, \\
& s_{q}=8 \frac{\left(2-s_{\nu}\right)}{\left(8-s_{\nu}\right)}
\end{aligned}
$$

where $\nu$ is the shear viscosity. The TRT model ensures accurate flow boundary conditions as well as adequate numerical stability [26, 27, 28, 4]. 
The equilibrium moments $\left\{m_{i}^{(0)} \mid i=0,1, \ldots, 8\right\}$ are given by

$$
\begin{array}{lll}
m_{0}^{(0)}=\delta \rho, & m_{1}^{(0)}=\rho_{0} u, & m_{2}^{(0)}=\rho_{0} v, \\
m_{3}^{(0)}=-2 \delta \rho+3 \rho_{0}\left(u^{2}+v^{2}\right), & m_{4}^{(0)}=\rho_{0}\left(u^{2}-v^{2}\right), & m_{5}^{(0)}=\rho_{0} u v, \\
m_{6}^{(0)}=-\rho_{0} u, & m_{7}^{(0)}=-\rho_{0} v, & \\
m_{8}^{(0)}=\delta \rho-3 \rho_{0}\left(u^{2}+v^{2}\right) . & &
\end{array}
$$

Note that the so-called incompressible approximation [29] has been used in the equilibria above, that is, it is assumed that $\rho=\rho_{0}+\delta \rho$ with $\rho_{0}=1$, and the nonlinear term $\delta \rho \boldsymbol{u}$ and higher order nonlinear terms have been neglected [29]. With the above equilibria, the speed of sound in the system is

$$
c_{s}=\frac{1}{\sqrt{3}} c .
$$

The kinematic viscosity coefficients $\nu$ and $\zeta$ of the system are:

$$
\nu=\zeta=\frac{1}{3}\left(\frac{1}{s_{\nu}}-\frac{1}{2}\right) .
$$

\subsection{D2Q5 model for the temperature}

We use the D2Q5 LB model to simulate the advection-diffusion equation for the temperature:

$$
\mathbf{g}\left(\boldsymbol{x}_{j}+\boldsymbol{c} \delta_{t}, t_{n}+\delta_{t}\right)=\mathbf{g}\left(\boldsymbol{x}_{j}, t_{n}\right)-\mathbf{N}^{-1} \cdot \mathbf{Q} \cdot\left[\mathbf{n}-\mathbf{n}^{(0)}\right]\left(\boldsymbol{x}_{j}, t_{n}\right),
$$

where the notations similar to that in Eq. (2) are used, i.e., bold-face font denotes 5-dimensional vectors. The discrete velocities in the D2Q5 model are the first five in the D2Q9 model, specified in Eq. (1). The temperature $T$ is the sole conserved quantity in the D2Q5 model and it is given by

$$
T=\sum_{i=0}^{4} g_{i} .
$$

The equilibrium moments $\left\{n_{i}^{(0)} \mid i=0,1, \ldots, 4\right\}$ are given by

$$
n_{0}^{(0)}=T, \quad n_{1}^{(0)}=u T, \quad n_{2}^{(0)}=v T, \quad n_{3}^{(0)}=a T, \quad n_{4}^{(0)}=0,
$$


where $u$ and $v$ are the velocity components obtained from the D2Q9 model for the flow fields, and $a \in(-4,1)$ is a parameter the effect of which is to be studied later in Sec. 5.1.

The transformation matrix $\mathrm{N}$ maps the distribution functions $\left\{g_{i} \mid i=\right.$ $0,1, \ldots, 4\}$ to the corresponding moments $\left\{n_{i} \mid i=0,1, \ldots, 4\right\}$ and is given by

$$
\mathbf{N}=\left(\begin{array}{ccccc}
1 & 1 & 1 & 1 & 1 \\
0 & 1 & 0 & -1 & 0 \\
0 & 0 & 1 & 0 & -1 \\
-4 & 1 & 1 & 1 & 1 \\
0 & 1 & -1 & 1 & -1
\end{array}\right)
$$

The diagonal relaxation matrix $Q$ is given by

$$
\mathbf{Q}=\operatorname{diag}\left(1, \sigma_{\kappa}, \sigma_{\kappa}, \sigma_{e}, \sigma_{\nu}\right) .
$$

The heat diffusivity $\kappa$ is given by

$$
\kappa=\frac{(4+a)}{10}\left(\frac{1}{\sigma_{\kappa}}-\frac{1}{2}\right) .
$$

To achieve the isotropy of the fourth-order error term in the D2Q5 model, it is required that

$$
\left(\frac{1}{\sigma_{\nu}}-\frac{1}{2}\right)\left(\frac{1}{\sigma_{\kappa}}-\frac{1}{2}\right)=\frac{1}{6} \quad \text { or } \quad \sigma_{\nu}=6 \frac{\left(2-\sigma_{\kappa}\right)}{\left(6-\sigma_{\kappa}\right)} .
$$

When the above relationship between $\sigma_{\kappa}$ and $\sigma_{\nu}$ is satisfied, one can eliminate the fourth order term in the equivalent equations for the D2Q5 model with the following relationship between $\sigma_{e}$ and $\sigma_{\kappa}$ [30]:

$$
\sigma_{e}=\frac{6(1-a)\left(2-\sigma_{\kappa}\right) \sigma_{\kappa}}{(7+3 a)\left(\sigma_{\kappa}-6\right) \sigma_{\kappa}+12(4+a)} .
$$

If and only if $\sigma_{\kappa}$ takes the following special value

$$
\frac{1}{\sigma_{\kappa}}=\frac{1}{2}+\frac{\sqrt{3}}{6},
$$


the relationship between $\sigma_{e}$ and $\sigma_{\kappa}$ is much simplified, consequently $\sigma_{e}$ and $\sigma_{\nu}$ become equal to the following value:

$$
\left(\frac{1}{\sigma_{e}}-\frac{1}{2}\right)=\left(\frac{1}{\sigma_{\nu}}-\frac{1}{2}\right)=\frac{\sqrt{3}}{3} .
$$

With $\sigma_{\kappa}$ fixed at the value specified by Eq. (19), $\kappa$ depends only on the parameter $a$ :

$$
\kappa=\frac{\sqrt{3}(4+a)}{60} .
$$

Thus, with the special value of $\sigma_{\kappa}$ given by Eq. (19) and $\sigma_{e}=\sigma_{\nu}$ given by Eq. (20), we have the D2Q5 TRT model [8, 9, 10, 11, 12], which eliminates the fourth order term in its equivalent equation for the temperature. Unless otherwise specified, we use the special value of $\sigma_{\kappa}$ given by Eq. (19) in our simulations.

\subsection{Macroscopic equations}

In incompressible thermal flows, thermal convection is due to the buoyancy effect induced by the density variation caused by the temperature variation. The density variation due to temperature change is approximated by the Boussinesq approximation:

$$
\rho=\rho_{0}\left[1+\alpha\left(T-T_{0}\right)\right]
$$

where $\rho_{0}$ is the reference density at the temperature $T_{0}, T_{0}$ is a reference temperature, and $\alpha$ is the thermal expansion coefficient under constant pressure:

$$
\alpha:=\left.\frac{1}{\rho_{0}} \frac{\partial \rho}{\partial T}\right|_{p} .
$$

With the Boussinesq approximation, the viscous heat dissipation and compression work due to pressure are neglected, and all the transport coefficients $(\nu$ and $\kappa$ ) and $\alpha$ are constants. The buoyancy force is therefore given by

$$
\boldsymbol{F}=\rho_{0} \alpha\left(T-T_{0}\right) g \hat{\boldsymbol{g}}
$$


where $g$ is the gravity and $\hat{\boldsymbol{g}}$ is the unit vector along the direction of the gravitational force.

The macroscopic equations derived from the D2Q9+D2Q5 LBE system are the following:

$$
\begin{aligned}
& \partial_{t} \boldsymbol{u}+\boldsymbol{u} \cdot \boldsymbol{\nabla} \boldsymbol{u}=-\frac{1}{\rho_{0}} \boldsymbol{\nabla} p+\nu \nabla^{2} \boldsymbol{u}+\alpha\left(T-T_{0}\right) g \hat{\boldsymbol{g}}, \\
& \boldsymbol{\nabla} \cdot \boldsymbol{u}=0, \\
& \partial_{t} T+\boldsymbol{u} \cdot \boldsymbol{\nabla} T=\kappa \nabla^{2} T .
\end{aligned}
$$

Equations (25b) and (25c) can be non-dimensionalized by choosing a characteristic length $L$ and temperature $T_{0}$, while the velocity is normalized by $\kappa / L$ and the pressure by $\rho_{0} c^{2}\left(c:=\delta_{x} / \delta_{t}\right)$ :

$$
\begin{aligned}
& \partial_{t} \boldsymbol{u}+\boldsymbol{u} \cdot \boldsymbol{\nabla} \boldsymbol{u}=-\boldsymbol{\nabla} p+\operatorname{Pr} \nabla^{2} \boldsymbol{u}+\operatorname{Ra} \operatorname{Pr} \theta \hat{\boldsymbol{g}}, \\
& \boldsymbol{\nabla} \cdot \boldsymbol{u}=0 \\
& \partial_{t} \theta+\boldsymbol{u} \cdot \boldsymbol{\nabla} \theta=\nabla^{2} \theta
\end{aligned}
$$

where $\theta:=\left(T-T_{0}\right) / T_{0}, \operatorname{Pr}:=\nu / \kappa$ is the Prandtl number which is fixed at the value of 0.71 (for air) unless otherwise specified, $\mathrm{Ra}=\mathrm{Pr} \cdot \mathrm{Gr}$ is the Rayleigh number, and Gr is the Grashof number:

$$
\mathrm{Gr}:=\frac{\alpha g \Delta T L^{3}}{\nu^{2}},
$$

where $\Delta T$ is the reference temperature difference. The thermal diffusivity $\kappa$ and the shear viscosity $\nu$ can also be given by

$$
\kappa=\sqrt{\frac{\alpha g \Delta T L^{3}}{\operatorname{Pr} \cdot \operatorname{Ra}}}, \quad \nu=\operatorname{Pr} \cdot \kappa .
$$

The characteristic velocity due to thermal convection is

$$
U=\sqrt{\alpha g \Delta T L}=\sqrt{\frac{\operatorname{Ra}}{\operatorname{Pr}}} \frac{\nu}{L} .
$$

The Mach number Ma based on the thermal convective velocity $U$ has to be kept small enough in order to maintain the stability criterion for the LBE, 
which can be expressed as an upper bound for the viscosity $\nu$ :

$$
\nu<\frac{\mathrm{Ma}^{*}}{\sqrt{3}} \sqrt{\frac{\mathrm{Pr}}{\mathrm{Ra}}} L,
$$

where $\mathrm{Ma}^{*}$ is the critical Mach number. If we choose $\mathrm{Ma}^{*} \approx 0.3$ and the minimum length $L \approx 13 \delta_{x}$, then the upper bound for $\nu$ is given by

$$
\nu<\min \left[\frac{\mathrm{Ma}}{\sqrt{3}} \sqrt{\frac{\mathrm{Pr}}{\mathrm{Ra}}} L, \sqrt{\frac{3.6}{\mathrm{Ra}}}\right] .
$$

The upper bound of $\nu$ is particularly important when $L$ is small. Note that for small Prandtl number, the thermal diffusivity $\kappa$ may become so large that $a>1$ in Eq. (21), thus violating the stability condition of the D2Q5 model.

In the LBE, the Mach number Ma plays the role of Courant-FriedrichsLewy (CFL) number. It has been shown [4] that for the D2Q9+D2Q5 TLBE for incompressible thermal flows, the Mach number Ma determines dimensionless time-step size, hence the computational efficiency, but it has little effect on the accuracy of results.

In what follows, we will use $\mathrm{Ma}=0.1$ in our simulations unless otherwise specified. The upper bound for viscosity $\nu$ given by Eq. (32) is used to ensure the numerical stability of the simulations especially when small grid sizes are used. Note that the transport coefficients in the LBE are Mach-number Ma dependent and the $\mathrm{Ma}^{2}$ terms are non-isotropic, i.e., they depend on the directly with respect to the lattice line [7]. These defects can be eliminated using LB models with more discrete velocities [31, 32]. However, tests show that the LB models with more discrete velocities make little difference on integrated quantities such as Nusselt numbers. We therefore use the D2Q5 model for the sake of computational efficiency.

\subsection{Implementation of the $L B$ algorithm}

We provide a step-by-step implementation of the MRT-LB algorithm as the following: 
1. Compute the conserved quantities $\delta \rho$ and $\boldsymbol{u}$ from $\left\{f_{i}\right\}$, and $T$ from $\left\{g_{i}\right\}$

2. Compute the force of Eq. (24) with the local temperature $T\left(\boldsymbol{x}_{j}, t_{n}\right)$;

3. Update the velocity: $\boldsymbol{u}^{*}=\boldsymbol{u}+\boldsymbol{F} \delta_{t} / 2 \rho_{0}$;

4. Compute the equilibrium moments $\left\{m_{i}^{(0)}\right\}$ with $\delta \rho$ and $\boldsymbol{u}^{*}$, and $\left\{n_{i}^{(0)}\right\}$ with $T$;

5. Compute the changes of the nonequilibrium moments $\left\{m_{i} \mid i=3, \ldots, 8\right\}$ for the D2Q9 model and that for the D2Q5 model, i.e., $\delta \mathbf{m}=-\mathrm{S} \cdot[\mathbf{m}-$ $\left.\mathbf{m}^{(0)}\right]$ and $\delta \mathbf{n}=-\mathbf{Q} \cdot\left[\mathbf{n}-\mathbf{n}^{(0)}\right]$;

6. Update the velocity again: $\boldsymbol{u}^{* *}=\boldsymbol{u}^{*}+\boldsymbol{F} \delta_{t} / 2 \rho_{0}=\boldsymbol{u}+\boldsymbol{F} \delta_{t} / \rho_{0}$;

7. Compute the changes of the distribution functions due to collisions and forcing, i.e., $\delta \mathbf{f}=\mathbf{M}^{-1} \cdot \delta \mathbf{m}$ and $\delta \mathbf{g}=\mathbf{N}^{-1} \cdot \delta \mathbf{n}$, then compute the post-collision distributions: $\mathbf{f}^{*}=\mathbf{f}+\delta \mathbf{f}$ and $\mathbf{g}^{*}=\mathbf{g}+\delta \mathbf{g}$ in the fluid nodes;

8. Advect $\left\{f_{i}\right\}$ and $\left\{g_{i}\right\}$, with special rules at boundaries.

A few remarks are in order here. First, the forcing term $\boldsymbol{F}$ is split into two halves according to Strang splitting so a second-order accuracy can be achieved (see the proof in [33]). Secondly, the MRT collision model is based on the projection method. The forcing term $\boldsymbol{F}$ is correctly projected on whatever modes (or moments) which the force is supposed to affect directly. This implementation is thus free of the numerical artifacts generated by the force term implemented in the discrete velocity space, i.e., $\left\{f_{i}\right\}$, in the LBGK schemes [34]. And thirdly, the collision and the advection steps, i.e., steps 7 and 8 in the procedure described above, should be combined together in one step in order to improve computational efficiency. If the collision and advection are executed separately in two steps, then the same data will have to be read and written into memory twice, which can severely degrade the computational efficiency due to unnecessary memory I/O. This problem can be rather severe when the data array size is so large that it cannot fit in cache. 


\section{Two-dimensional square cavity with differentially heated verti- cal walls}

\subsection{Flow configuration}

The flow configuration for the 2D square cavity with differentially heated vertical walls is shown in Fig. 1(left). The dimension of the cavity is $L \times H$. In this work, we set $L=H$. The left and right vertical walls of the cavity are kept at constant temperatures $\theta_{\mathrm{h}}=+0.5$ and $\theta_{\mathrm{c}}=-0.5$, respectively, while the top and bottom walls are adiabatic. All walls are impenetrable, rigid and no-slip. The system is subject to gravity $\hat{\boldsymbol{g}}$ with the downward direction $-\hat{\boldsymbol{y}}$, as shown in Fig. 1(left). In the normalized coordinates $(x, y) \in \Omega:=$ $[0,1] \times[0,1]$, the boundary conditions are given by:

$$
\begin{aligned}
& \left.\boldsymbol{u}\right|_{x \in \partial \Omega}=\mathbf{0}, \\
& \left.\theta\right|_{x=0}=+0.5,\left.\quad \theta\right|_{x=1}=-0.5, \\
& \left.\partial_{\hat{\boldsymbol{n}}} \theta\right|_{y=0}=\left.\partial_{\hat{\boldsymbol{n}}} \theta\right|_{y=1}=0,
\end{aligned}
$$

where $\hat{\boldsymbol{n}}$ is the unit out-normal vector to the boundary $\partial \Omega$.

The initial state of the flow is quiescent and isothermal, i.e., $\boldsymbol{u}\left(\boldsymbol{x}_{j}, t_{0}\right)=$ $\mathbf{0}, \delta \rho\left(\boldsymbol{x}_{j}, t_{0}\right)=0$, and $\theta\left(\boldsymbol{x}_{j}, t_{0}\right)=0$ for all $\boldsymbol{x}_{j}$. The Prandtl number is fixed at $\operatorname{Pr}=0.71$ (for air), and the values of the Rayleigh number are $\mathrm{Ra}=10^{6}$, $10^{7}$, and $10^{8}$. The flow remains time-independent under these conditions (cf., e.g., $[15])$.

We use meshes of uniform Cartesian grid of size $\left(N_{x}+2\right) \times\left(N_{y}+2\right)$ for our simulations, as illustrated in Fig. 1(right). The flow domain $L \times H$ is covered by the fluid nodes $(i, j) \in\left[1, N_{x}\right] \times[1, N y]$. The flow domain is wrapped with a buffer layer, i.e., $i=0$ and $i=\left(N_{x}+1\right), j=0$, and $j=\left(N_{y}+1\right)$, as shown in Fig. 1(right), for the convenience of storing the outgoing distribution functions during the advection step.

\subsection{Boundary Conditions}

For the velocity field $\boldsymbol{u}$, only the no-slip boundary conditions are used for all four walls of the cavity. The no-slip boundary conditions can be realized 

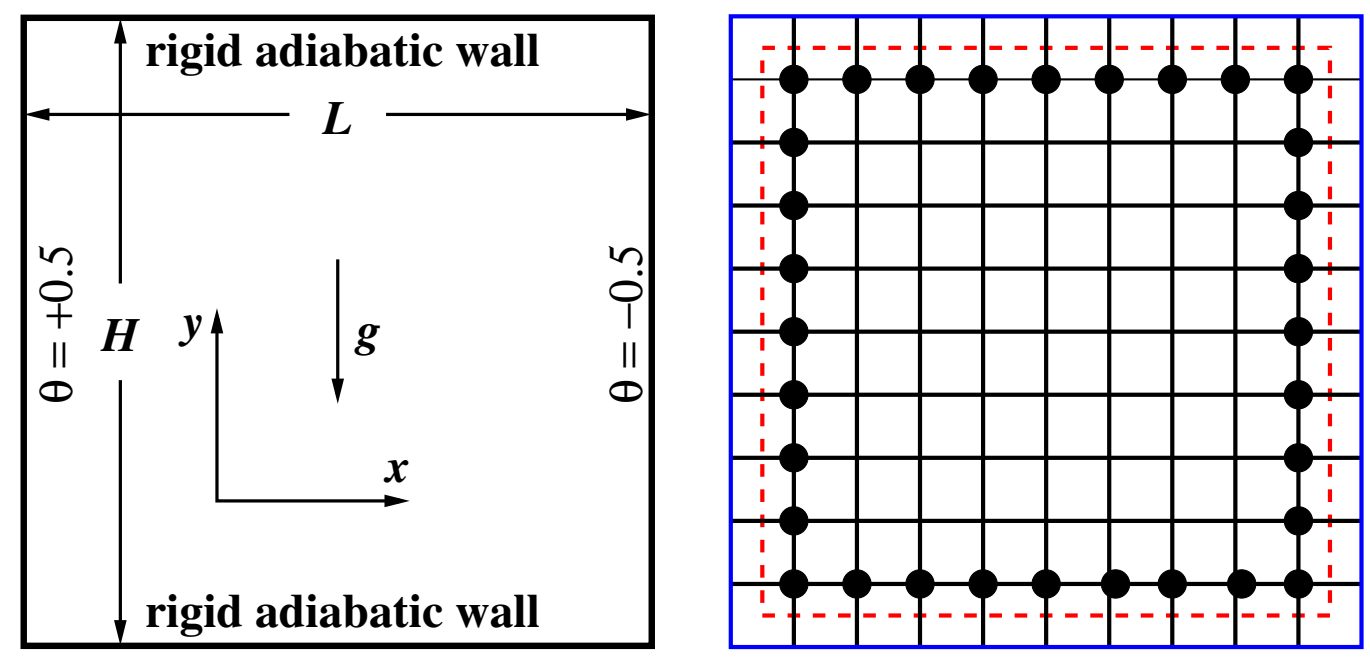

Figure 1: (Left) A schematic of the 2D square cavity of dimension $L \times H$ with differentially heated vertical walls and other boundary conditions. (Right) A schematic of a mesh: the boundary nodes are marked with solid discs $\bullet$, the boundary conditions are satisfied on the dashed line, nodes outside the dashed line are used as buffer layer.

by the bounce-back (BB) boundary conditions, that is, when a particle hits the wall, it reverses its momentum as the following:

$$
f_{\bar{\imath}}\left(\boldsymbol{x}_{\mathrm{B}}, t_{n}+\delta_{t}\right)=f_{i}^{*}\left(\boldsymbol{x}_{\mathrm{B}}, t_{n}\right)
$$

where $\boldsymbol{x}_{\mathrm{B}}$ is a fluid node adjacent to a boundary, i.e., a boundary node (indicated by $\bullet$ in Fig. 1(right)), $f_{\bar{\imath}}$ corresponds to $\boldsymbol{c}_{\bar{\imath}}:=-\boldsymbol{c}_{i}, f_{i}^{*}\left(\boldsymbol{x}_{\mathrm{B}}, t_{n}\right)$ denotes the post collision value of the distribution function. The bounceback boundary conditions are applied to the distributions in the directions not parallel to a wall.

As for the temperature field $\theta$ (or $T$ ), there are two types of boundary conditions, i.e., that of constant temperature and of adiabatic. The adiabatic boundary conditions are Neumann boundary conditions for $\theta$ (zero heat fluxes) which can be realized by the bounce-back boundary conditions for the distributions $g_{i}$, as prescribed by Eq. (35). The temperature boundary conditions are the Dirichlet boundary conditions for $\theta$ which can be realized 
by the anti-bounce-back boundary conditions:

$$
g_{\bar{\imath}}\left(\boldsymbol{x}_{\mathrm{B}}, t_{n}+\delta_{t}\right)=-g_{i}^{*}\left(\boldsymbol{x}_{\mathrm{B}}, t_{n}\right)+\frac{4+a}{10} \theta_{\mathrm{w}},
$$

where $\theta_{\mathrm{w}}$ is the wall temperature. It should be noted that as far as $\theta$ is concerned, the four corners of the square belong to the vertical walls. This, of course, is an arbitrary choice, for they also belong to the top and bottom boundaries.

We would like to emphasize that the MRT-LBE (or TRT-LBE) is imperative to achieve accurate and consistent boundary conditions for both $\boldsymbol{u}$ and $\theta$ imposed exactly at the same location, i.e., one-half grid spacing, $\delta_{x} / 2$, beyond the last fluid nodes (or the boundary nodes) for straight boundaries parallel to lattice lines, such is the case for the cavity flow $[9,4]$. For the lattice Bhatnagar-Gross-Krook (LBGK) models, the exact boundary location depends on both relaxation parameters for $f_{i}$ and $g_{i}$, so its results depend on the grid size and cannot converge [26, 28].

\section{Post processing and measurements}

Various averaged and local quantities are measured in order to gauge the quality of simulations and different numerical methods can be compared. We are only concerned with the steady state solutions of the problem. To achieve a steady state, the following criteria are used in our simulations [4]:

$$
\begin{gathered}
\frac{\sum_{j}\left\|\boldsymbol{u}\left(\boldsymbol{x}_{j}, t_{n+1000}\right)-\boldsymbol{u}\left(\boldsymbol{x}_{j}, t_{n}\right)\right\|_{2}}{\sum_{j}\left\|\boldsymbol{u}\left(\boldsymbol{x}_{j}, t_{n+1000}\right)\right\|_{2}}<10^{-12}, \\
\left\|\theta\left(\boldsymbol{x}_{j}, t_{n+1000}\right)-\theta\left(\boldsymbol{x}_{j}, t_{n}\right)\right\|_{\infty}<10^{-6},
\end{gathered}
$$

where $\|\cdot\|_{2}$ and $\|\cdot\|_{\infty}$ denote the Euclidean $L_{2}$ norm and $L_{\infty}$ norm, respectively.

For the steady convective flow in the 2D square with differentially heated vertical walls, the averaged quantities of interest are various Nusselt numbers. Of the interest there are also some local hydrodynamic quantities and their precise locations. We will provide a succinct description of measuring these quantities in what follows. 


\subsection{Nusselt numbers}

The local heat flux in the horizontal direction $\hat{\boldsymbol{x}}$ between two differentially heated vertical walls is given by

$$
q_{x}=u \theta-\partial_{x} \theta,
$$

which will be used to compute various Nusselt numbers. We will compute three Nusselt numbers: one averaged over the entire flow domain, one averaged over left wall at $x=0$, and one averaged over the vertical symmetric line of the cavity, denoted by $\langle\mathrm{Nu}\rangle,\langle\mathrm{Nu}\rangle_{0}$, and $\langle\mathrm{Nu}\rangle_{1 / 2}$, respectively. The volume average Nusselt number $\langle\mathrm{Nu}\rangle$ is given by

$$
\langle\mathrm{Nu}\rangle=\frac{1}{L H \Delta \theta} \int_{0}^{L} \int_{0}^{H} q_{x} \mathrm{~d} x \mathrm{~d} y \approx \frac{1}{N_{x} N_{y} \Delta \theta} \sum_{i, j=1}^{N_{x}, N_{y}} q_{x}(i, j),
$$

where $\Delta \theta:=\theta(x=0)-\theta(x=1)=1$. Similarly, $\langle\mathrm{Nu}\rangle_{0}$ and $\langle\mathrm{Nu}\rangle_{1 / 2}$ are computed as the following:

$$
\begin{gathered}
\langle\mathrm{Nu}\rangle_{0} \approx \frac{1}{N_{y} \Delta \theta} \sum_{j=1}^{N_{y}} q_{x}(x=0, j), \\
\langle\mathrm{Nu}\rangle_{1 / 2} \approx \frac{1}{N_{y} \Delta \theta} \sum_{j=1}^{N_{y}} q_{x}(x=1 / 2, j) .
\end{gathered}
$$

In theory, all three Nusselt numbers should be equal. Thus, differences among them measure the numerical inconsistency in the energy conservation.

Except for the boundary nodes, the heat flux is computed by the following central-differencing formula

$$
q_{x}(i, j)=\frac{\theta(i+1, j)-\theta(i-1, j)}{2 \delta_{x}}, \quad 1<i<N_{x}, 1 \leq j \leq N_{y} .
$$

For the boundary nodes at $i=1$, the heat flux is approximated by:

$$
q_{x}(i, j)=\frac{\theta(i+1, j)+\theta(i, j)-2 \theta_{\mathrm{h}}}{2 \delta_{x}}, \quad i=1,1 \leq j \leq N_{y},
$$


where $\theta_{\mathrm{h}}:=\left.\theta\right|_{x=0}=0.5$ is the temperature at the left wall. The heat flux at $i=N_{x}$ can evaluated similarly.

To obtain $\langle\mathrm{Nu}\rangle_{0}$, we need to compute $q_{x}$ at $x=0$ as the following:

$$
q_{x}(x=0, j)=\frac{2\left[\theta(1, j)-\theta_{\mathrm{h}}\right]}{\delta_{x}}, \quad 1 \leq j \leq N_{y} .
$$

To compute $q_{x}$ at two corners on the left, i.e., $(x, y)=(0,0)$ and $(x, y)=$ $(0,1)$, the temperature at the horizontal walls $y=0$ and $y=1$ are evaluated by using four points along each vertical lattice line and nearest to the horizontal walls to fit the following temperature profile [4] with the least-square method:

$$
\theta(y)=c_{0}+c_{2}\left(y-y_{0}\right)^{2},
$$

where $y_{0}=0$ (or 1 ) is the position of the bottom (or top) horizontal wall. Then $q_{x}$ along two horizontal walls can be evaluated according to Eq. (44).

We also compute the maximum and minimum Nusselt numbers $\mathrm{Nu}_{\max }$ and $\mathrm{Nu}_{\text {min }}$ at the hot wall on the left $(x=0)$. To find $\mathrm{Nu}_{\max }$, we first identify the maximum value of $\mathrm{Nu}$ on a grid, then fit the values of $\mathrm{Nu}$ on five points about the grid with a parabola by using least-square method. The fitted parabola is then used to interpolate $\mathrm{Nu}_{\max }$ and its position on the left wall.

As for $\mathrm{Nu}_{\text {min }}$, since its location is always very close to the top left corner (in fact results converge towards the top left corner), only grid points located before the minimum value are used to find the interpolating polynomial.

\subsection{Hydrodynamic intensities}

The hydrodynamic quantities of interest include the value of the streamfunction at the cavity center $\psi_{\text {mid }}$, the maximum magnitude of the streamfunction $|\psi|_{\max }$ and its location, the maximum $x$-component of the velocity on the vertical center-line $u_{\max }$, and the maximum $y$-component of the velocity on the horizontal center-line $v_{\max }$. The maximum velocities and their positions are calculated in the same way as the maximum Nusselt number $\mathrm{Nu}_{\max }$ is done. 
We also compute the maximum of the stream function $\psi$ and its value at the center of the cavity. The stream-function is defined as

$$
\psi(x, y)=\int_{0}^{y} u(x, \mu) \mathrm{d} \mu=-\int_{0}^{x} v(\eta, y) \mathrm{d} \eta
$$

The integral is approximated by the third-order Simpson's rule of cumulative summation using second-order polynomial interpolation coefficients. The maximum magnitude of the stream-function, $|\psi|_{\max }$, is obtained on a fine mesh of $10001 \times 10001$ equidistant points, as in [15]. The values of $\psi$ on this fine mesh are obtained by using a cubic spline interpolation of the LB data on coarser meshes.

\section{The results}

The LB algorithm has been implemented on both CPU and GPU. With a mesh of size $N^{2}=2043^{2}$, the LB code running on an Intel Core-I7 CPU and on a Quadro-6000 GPU can achieve about, respectively, 12 and 1080 millions site updates per second in single precision and 9 and 560 millions per second in double precision. For a smaller mesh size of $256^{2}$, the speed on the Quadro-6000 GPU is 396 and 617 millions site updates per second in single and double precision, respective. The GPU allows us to obtain the results of steady-state with a mesh size as large as $2043^{2}$ within 15 hours.

The simulations are conducted with the following formula to determine the parameter $a$, which is obtained by using $\kappa=\nu / \operatorname{Pr}$, the special value of $\sigma_{\kappa}$ given by Eq. (19), and the upper bound of $\nu$ given by Eq. (32):

$$
a \leq \frac{20 \cdot \mathrm{Ma} \cdot N}{\sqrt{\mathrm{Pr} \cdot \mathrm{Ra}}}-4,
$$

with the condition $-4<a<1$ for thermal stability - The lower bound -4 corresponds to a positivity of $\kappa$ in $\mathrm{Eq}(21)$ and the upper bound +1 corresponds to stability limit of the checkers-board mode. In our simulations, the Mach number $\mathrm{Ma}$ is fixed at 0.1 unless otherwise specified, and $\mathrm{Pr}=$ 0.71 , the equality usually holds. Thus, with the Rayleigh number Ra and 
the resolution $N$ given, the parameter $a$ is determined, and so are the heat diffusivity $\kappa$ and the viscosity $(\nu=\operatorname{Pr} \kappa)$, the value of $\nu$ is also checked against the stability criterion of Eq. (33).

\subsection{Effect of the parameter a}

The parameter $a$, which determines the heat diffusivity $\kappa$ [cf. Eqs. (16) and (21)], may affect the numerical stability, among other things. It is clear from Eq. (47) that the parameter $a$ has to be a variable: the Prandtl number Pr is fixed, the Mach number Ma is chosen in consideration of computational efficiency [4], and with a given Rayleigh number Ra, the parameter $a$ varies with the grid resolution $N$. It has been observed that the main effect of the parameter $a$ is on the magnitude of the spatial oscillations of the heat flux $q_{x}$ near boundaries [4]. The quantify the effect due to $a$, we compute the Nusselt number $\langle\mathrm{Nu}\rangle_{y}(x)$ averaged over $y$ along each grid line, similar to the way $\langle\mathrm{Nu}\rangle_{0}$ and $\langle\mathrm{Nu}\rangle_{1 / 2}$ are computed, and as a function of $x$. We then compute the maximum oscillation magnitude, $\max \left|\delta\langle\mathrm{Nu}\rangle_{y}\right|$, of $\langle\mathrm{Nu}\rangle_{y}(x)$ as a function of $a$, with given Ra and resolution $N^{2}$.

To conduct a parametric study on the effect of $a$, we cannot use the equality of Eq. (47) for $a$, that is, we cannot fix $\sigma_{\kappa}$ with the value of Eq. (19). Instead, with Eqs. (16) and (30), we have

$$
a=\frac{10 \cdot N}{\sqrt{\mathrm{Pr} \cdot \mathrm{Ra}}}\left(\frac{1}{\sigma_{\kappa}}-\frac{1}{2}\right)^{-1}-4=\frac{20 \cdot N}{\sqrt{\mathrm{Pr} \cdot \mathrm{Ra}}} \frac{\sigma_{\kappa}}{\left(2-\sigma_{\kappa}\right)}-4 .
$$

Thus, with $\operatorname{Pr}=0.71$ fixed, and $N$ and Ra given, we can adjust $a$ by varying $\sigma_{\kappa} \in(0,2)$. In Fig. 2 we show $\max \left(\delta\langle\mathrm{Nu}\rangle_{y}\right)$ as a function of $a$ with $\mathrm{Ra}=$ $10^{7}$ and two different resolutions $N^{2}=251^{2}$ and $379^{2}$. It is clearly seen from Fig. 2 that, independent of the resolution $N^{2}$, the maximum oscillation magnitude $\max \left|\delta\langle\mathrm{Nu}\rangle_{y}\right|$ monotonically decreases as $a$ decreases from 1 to reach a minimum at about $a \approx 3.90$, then rapidly increases as $a$ approaches to -4 - the stability limit at $\kappa=0$. It should be noted that the accuracy of the LBE is not affected by the value of $a$, so long as the LBE is stable. 


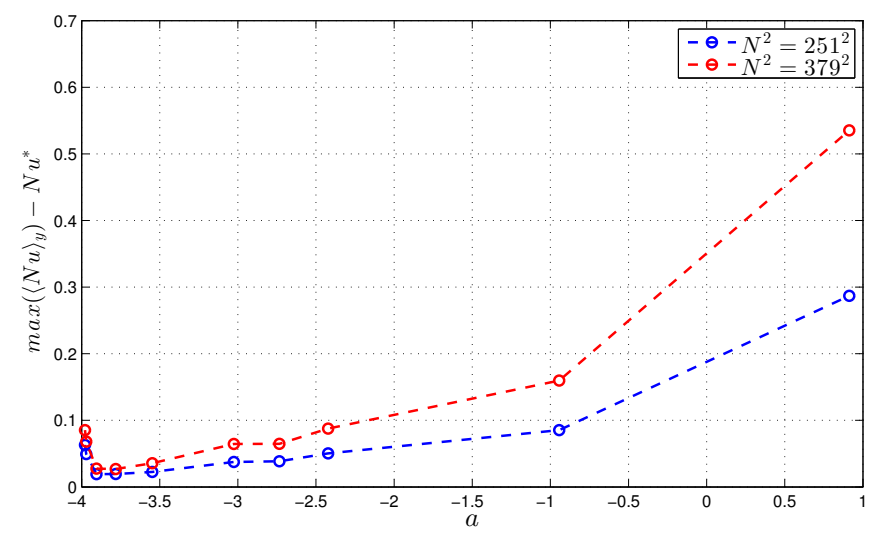

Figure 2: The maximum oscillation magnitude of $\langle\mathrm{Nu}\rangle_{y}, \max \left|\delta\langle\mathrm{Nu}\rangle_{y}\right|$, as a function of the parameter $a$. Ma $=0.1, R a=10^{7}, N^{2}=251^{2}$ and $379^{2}$.

\subsection{Global convergence of flow fields}

We conduct a convergence study by using a number of mesh sizes $N_{x} \times$ $N_{y}=N^{2}\left(N_{x}=N_{y}=N\right)$ between $251^{2}$ and $2043^{2}$. We compute the $L_{2}$ errors in the velocity $\boldsymbol{u}$ and the temperature $\theta$ as the following:

$$
\begin{aligned}
\|\delta \boldsymbol{u}\|_{2} & :=\frac{\sum_{j}\left\|\boldsymbol{u}\left(\boldsymbol{x}_{j}\right)-\boldsymbol{u}^{*}\left(\boldsymbol{x}_{j}\right)\right\|_{2}}{\sum_{j}\left\|\boldsymbol{u}^{*}\left(\boldsymbol{x}_{j}\right)\right\|_{2}}, \\
\|\delta \theta\|_{2} & :=\frac{\sum_{j}\left\|\theta\left(\boldsymbol{x}_{j}\right)-\theta^{*}\left(\boldsymbol{x}_{j}\right)\right\|_{2}}{\sum_{j}\left\|\theta^{*}\left(\boldsymbol{x}_{j}\right)\right\|_{2}}
\end{aligned}
$$

where the reference solutions $\boldsymbol{u}^{*}\left(\boldsymbol{x}_{j}\right)$ and $\theta^{*}\left(\boldsymbol{x}_{j}\right)$ are obtained with the largest mesh of the size $N^{2}=2043^{2}$.

For simulations with mesh sizes $N^{2} \geq 763^{2}$ which are carried out on a GPU, we use the following values for the relaxation rates: $s_{e}=1.20$, $s_{\epsilon}=1.30, s_{q}$ given by Eq. (7b), and the values of $s_{\nu}$ are given in Table 1. The values given in Table 1 satisfy the stability condition of Eq. (32).

Because the boundary conditions for both $\boldsymbol{u}$ and $\theta$ are satisfied at the locations $\delta_{x} / 2$ beyond the last fluid nodes, the meshes of different sizes have no overlapping grid points except the boundaries. To compute the differences of the flow fields on difference meshes, we interpolate the data on the finest 


\begin{tabular}{l|l|l|l}
\hline \multicolumn{1}{r|}{$\mathrm{Ra}$} & $10^{6}$ & $10^{7}$ & $10^{8}$ \\
\hline \hline $1019^{2}$ & 0.050 & 0.050 & 0.025 \\
\hline $1531^{2}$ & 0.050 & 0.050 & 0.025 \\
\hline $2043^{2}$ & 0.070 & 0.070 & 0.035 \\
\hline
\end{tabular}

Table 1: The viscosity $\nu$ depending on the mesh size $N^{2}$ and the Rayleigh number Ra.

mesh to coarser meshes by using a cubic spline interpolation. The errors and the corresponding the order of accuracy $n$ are summarized in Tab. 2. If no interpolation is performed, i.e., the closest points in the meshes are used to compute the errors, the order of accuracy would appear to be significantly lower [4].

\begin{tabular}{c|l|l|l}
\hline Ra & \multicolumn{1}{|c}{$10^{6}$} & \multicolumn{1}{|c}{$10^{7}$} & \multicolumn{1}{|c}{$\|\delta \boldsymbol{u}\|_{2}$} \\
\hline \hline$N^{2}$ & \multicolumn{3}{|c}{} \\
\hline $379^{2}$ & $1.6249 \cdot 10^{-2}$ & $2.7804 \cdot 10^{-2}$ & $7.1627 \cdot 10^{-2}$ \\
$507^{2}$ & $1.1210 \cdot 10^{-2}$ & $1.9019 \cdot 10^{-2}$ & $4.5857 \cdot 10^{-2}$ \\
$763^{2}$ & $6.2005 \cdot 10^{-3}$ & $1.0560 \cdot 10^{-2}$ & $2.3677 \cdot 10^{-2}$ \\
$1019^{2}$ & $3.7144 \cdot 10^{-3}$ & $6.2934 \cdot 10^{-3}$ & $1.3401 \cdot 10^{-2}$ \\
$1531^{2}$ & $1.2367 \cdot 10^{-3}$ & $2.0827 \cdot 10^{-3}$ & $4.1838 \cdot 10^{-3}$ \\
\hline$n$ & 1.7977 & 1.8339 & 1.9843 \\
\hline & \multicolumn{3}{|c}{$\|\delta \theta\|_{2}$} \\
\hline $379^{2}$ & $1.2298 \cdot 10^{-2}$ & $1.6245 \cdot 10^{-2}$ & $2.1559 \cdot 10^{-2}$ \\
$507^{2}$ & $8.4736 \cdot 10^{-3}$ & $1.1182 \cdot 10^{-2}$ & $1.4804 \cdot 10^{-2}$ \\
$763^{2}$ & $4.6861 \cdot 10^{-3}$ & $6.1771 \cdot 10^{-3}$ & $8.1582 \cdot 10^{-3}$ \\
$1019^{2}$ & $2.8052 \cdot 10^{-3}$ & $3.6958 \cdot 10^{-3}$ & $4.8752 \cdot 10^{-3}$ \\
$1531^{2}$ & $9.3279 \cdot 10^{-4}$ & $1.2284 \cdot 10^{-3}$ & $1.6186 \cdot 10^{-3}$ \\
\hline$n$ & 1.8000 & 1.8022 & 1.8073 \\
\hline
\end{tabular}

Table 2: Global convergence of the velocity $\boldsymbol{u}$ and the temperature $\theta$ with the $L_{2}$-norm. $\mathrm{Ma}=0.1$. 
The order of accuracy, $n$, is about 1.8 or better for all cases in Tab. 2, indicating that the method is indeed second-order accurate. We also observe that the $L_{2}$ errors of $\boldsymbol{u}$ and $\theta$ increase with the Rayleigh number Ra. In fact, the data in Tab. 2 show that

$$
\ln \|\delta \boldsymbol{u}\|_{2} \propto \mathrm{Ra}^{1+\varepsilon}, \quad\|\delta \theta\|_{2} \propto \mathrm{Ra},
$$

where $\varepsilon>0$ depends on $N$. The above scalings corroborate previous results [4].

The contours of the stream function $\psi$, the temperature $\theta$, the pressure $p$, and the vorticity $\omega$ obtained with the finest mesh of $N^{2}=2043^{2}$ are shown in Fig. 3. The vorticity $\omega:=\left(\partial_{x} v-\partial_{y} u\right)$ is computed with a second-order central finite-difference scheme. The figures show the changes of flow features as the Rayleigh number Ra increases. Clearly the contours are antisymmetric about either the vertical or the horizontal center lines.

\subsection{Convergence of Nusselt numbers}

Table 3 shows the convergence behavior of the Nusselt numbers. The asymptotic values of the Nusselt numbers are obtained by using the leastsquare fitting with the cubic polynomial

$$
f(h)=f_{\infty}+a_{2} h^{2}+a_{3} h^{3},
$$

where $h=1 / N$ is the grid spacing, and $f_{\infty}$ is the asymptotic value for $h \rightarrow 0$, i.e., $N \rightarrow \infty$. The asymptotic values of Nu's are the reference values to compute the errors of Nu's, which are then used to compute the order of accuracy $n$. Both the asymptotic values and the order of accuracy $n$ are given in Tab. 3.

All the averaged Nusselt numbers, including $\langle\mathrm{Nu}\rangle,\langle\mathrm{Nu}\rangle_{0}$, and $\langle\mathrm{Nu}\rangle_{1 / 2}$, clearly achieve a second-order accuracy. It is also noticed that $\langle\mathrm{Nu}\rangle$ and $\langle\mathrm{Nu}\rangle_{1 / 2}$ agree with each other better than their agreement with $\langle\mathrm{Nu}\rangle_{0}$. This is understandable because $\langle\mathrm{Nu}\rangle_{0}$ is evaluated with a less accurate differencing formula [cf. Eq. (42) vs. Eq. (43)]. As for the local Nusselt numbers $\mathrm{Nu}_{\max }$ 

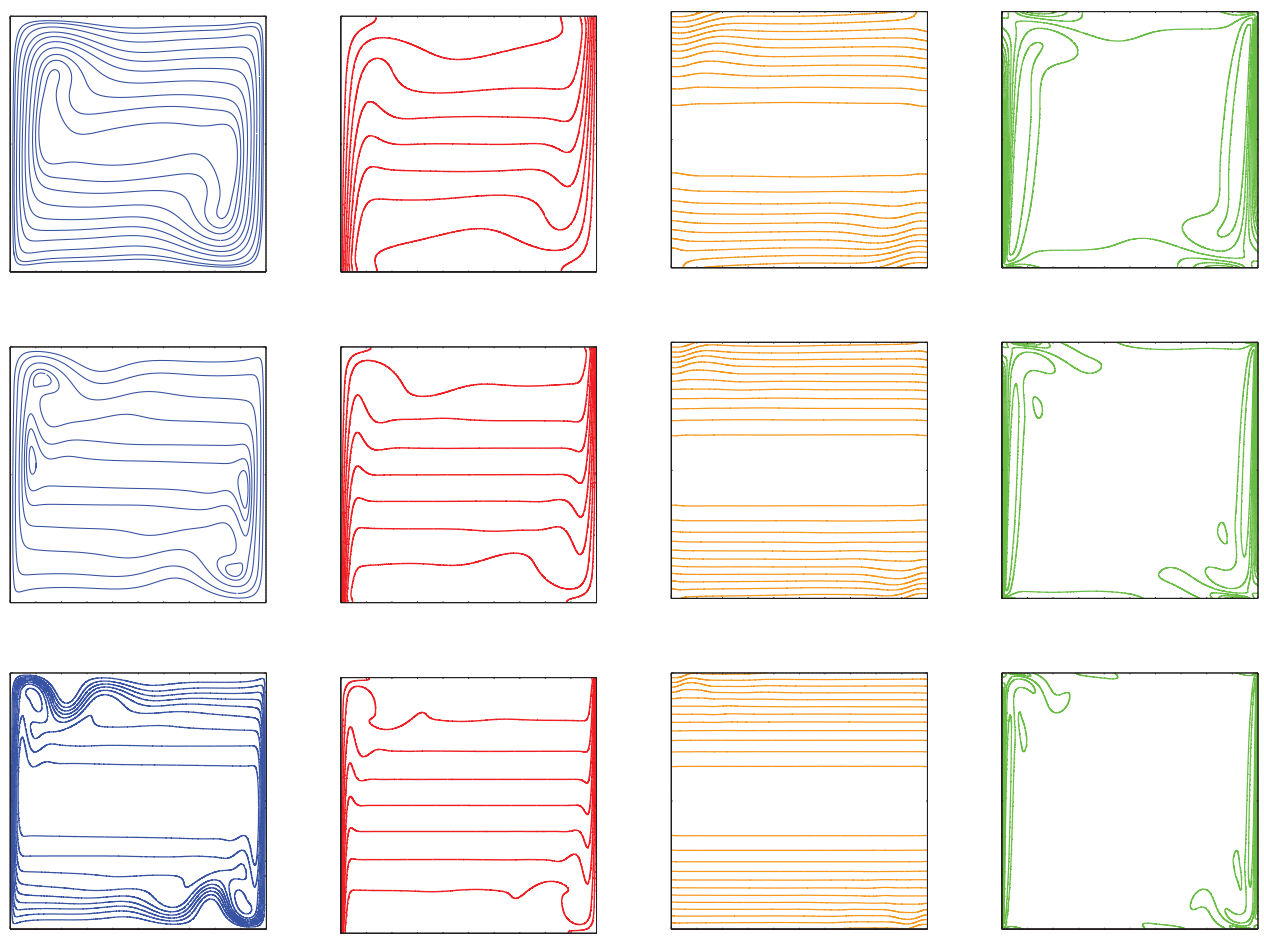

Figure 3: Contours of flow fields. From left to right: streamlines $(\psi)$, isotherms $(\theta)$, isobars $(p)$, and the vorticity contours $(\omega)$. from top to bottom: $\mathrm{Ra}=10^{6}, 10^{7}$, and $10^{8}$. $N=2043^{2}, \mathrm{Ma}=0.1$.

and $\mathrm{Nu}_{\text {min }}$ and their locations on the left wall, the accuracy is nominally second-order with $L_{\infty}$-norm, which is a much more stringent measure than $L_{2}$-norm. The worst case is the location of $\mathrm{Nu}_{\max }$, which becomes closer and closer to the lower-left corner of the cavity as Ra increases. It is clearly the case that the meshes are not fine enough to sufficiently resolve the region of high gradient near the vertical walls, especially at the lower-left and upperright corners (cf. Fig. 3).

To further investigate possible sources of error in computing the local Nusselt numbers, in Fig. 4 we show the $y$ variation of the heat flux on the left (hot) wall, $q_{x}(x=0, y)$, and the $x$ variation of the Nusselt number averaged over $y$ along each grid line, i.e., $\langle\mathrm{Nu}\rangle_{y}(x)$. We only show $\langle\mathrm{Nu}\rangle_{y}(x)$ 


\begin{tabular}{|c|c|c|c|c|c|c|c|c|}
\hline $\mathrm{Ra}$ & $N^{2}$ & $\langle\mathrm{Nu}\rangle$ & $\langle\mathrm{Nu}\rangle_{0}$ & $\langle\mathrm{Nu}\rangle_{1 / 2}$ & $\mathrm{Nu}_{\max }$ & $y$ & $\mathrm{Nu}_{\min }$ & $y$ \\
\hline \multirow{9}{*}{$10^{6}$} & $251^{2}$ & 8.8231 & 8.8288 & 8.8253 & 17.6252 & 0.0386 & 0.9774 & 0.9986 \\
\hline & $379^{2}$ & 8.8243 & 8.8265 & 8.8252 & 17.5802 & 0.0388 & 0.9795 & 0.9972 \\
\hline & $507^{2}$ & 8.8246 & 8.8258 & 8.8252 & 17.5622 & 0.0390 & 0.9794 & 0.9978 \\
\hline & $763^{2}$ & 8.8250 & 8.8255 & 8.8252 & 17.5482 & 0.0391 & 0.9794 & 0.9984 \\
\hline & $1019^{2}$ & 8.8251 & 8.8253 & 8.8252 & 17.5430 & 0.0391 & 0.9794 & 0.9987 \\
\hline & $1531^{2}$ & 8.8251 & 8.8253 & 8.8252 & 17.5392 & 0.0392 & 0.9794 & 0.9991 \\
\hline & $2043^{2}$ & 8.8252 & 8.8252 & 8.8252 & 17.5378 & 0.0392 & 0.9794 & 0.9993 \\
\hline & $\infty$ & 8.8252 & 8.8252 & 8.8252 & 17.5360 & 0.0392 & 0.9795 & 0.9994 \\
\hline & $n$ & 1.9526 & 2.2612 & 2.9656 & 1.8743 & 1.7717 & 1.7464 & 1.8427 \\
\hline \multirow{9}{*}{$10^{7}$} & $251^{2}$ & 16.5229 & 16.5579 & 16.5286 & 39.9459 & 0.0176 & 1.4185 & 0.9965 \\
\hline & $379^{2}$ & 16.5229 & 16.5357 & 16.5255 & 39.7402 & 0.0175 & 1.3903 & 0.9952 \\
\hline & $507^{2}$ & 16.5229 & 16.5292 & 16.5244 & 39.6199 & 0.0176 & 1.3783 & 0.9964 \\
\hline & $763^{2}$ & 16.5230 & 16.5254 & 16.5237 & 39.5085 & 0.0178 & 1.3696 & 0.9979 \\
\hline & $1019^{2}$ & 16.5230 & 16.5242 & 16.5234 & 39.4628 & 0.0178 & 1.3679 & 0.9984 \\
\hline & $1531^{2}$ & 16.5231 & 16.5235 & 16.5232 & 39.4628 & 0.0179 & 1.3669 & 0.9989 \\
\hline & $2043^{2}$ & 16.5231 & 16.5233 & 16.5232 & 38.4132 & 0.0179 & 1.3666 & 0.9992 \\
\hline & $\infty$ & 16.5231 & 16.5230 & 16.5231 & 39.3950 & 0.0180 & 1.3659 & 0.9994 \\
\hline & $n$ & 1.9291 & 2.2244 & 2.0256 & 1.8066 & 1.6538 & 2.1473 & 1.8590 \\
\hline \multirow{8}{*}{$10^{8}$} & $379^{2}$ & 30.2412 & 30.3314 & 30.2573 & 88.5991 & 0.0084 & 2.2745 & 0.9925 \\
\hline & $507^{2}$ & 30.2337 & 30.2779 & 30.2428 & 88.5233 & 0.0082 & 2.1062 & 0.9946 \\
\hline & $763^{2}$ & 30.2287 & 30.2444 & 30.2328 & 88.0838 & 0.0081 & 1.9882 & 0.9964 \\
\hline & $1019^{2}$ & 30.2271 & 30.2345 & 30.2294 & 87.8022 & 0.0082 & 1.9551 & 0.9973 \\
\hline & $1531^{2}$ & 30.2259 & 30.2286 & 30.2269 & 87.5323 & 0.0083 & 1.9336 & 0.9981 \\
\hline & $2043^{2}$ & 30.2255 & 30.2268 & 30.2261 & 87.4178 & 0.0083 & 1.9265 & 0.9986 \\
\hline & $\infty$ & 30.2251 & 30.2241 & 30.2251 & 87.2454 & 1.9063 & 1.9195 & 0.9990 \\
\hline & $n$ & 2.0832 & 2.2076 & 2.0326 & 1.6819 & 1.4232 & 2.3431 & 1.6294 \\
\hline
\end{tabular}

Table 3: Convergence behavior of the averaged Nusselt numbers $\langle\mathrm{Nu}\rangle,\langle\mathrm{Nu}\rangle_{0}$, and $\langle\mathrm{Nu}\rangle_{1 / 2}$, and the local Nusselt numbers $\mathrm{Nu}_{\max }$ and $\mathrm{Nu}_{\min }$ with their vertical positions on the left (hot) wall. Note: the data that do not fit well with the interpolating polynomial have been excluded from the computation of the asymptotic values.

for $0 \leq x \leq 1 / 2$ because $\langle\mathrm{Nu}\rangle_{y}(x)$ is anti-symmetric about $x=1 / 2$. Clearly, as Ra increases, not only the maximum of $q_{x}$ increases, but also the thermal boundary layer, which varies as $\mathrm{Ra}^{-1 / 4}$, becomes thinner as the gradient $\partial_{x} \theta$ becomes steeper near the lower-left corner, as shown in the figures of $q_{x}$ in Fig. 4. The oscillation in $\langle\mathrm{Nu}\rangle_{y}(x)$ is clearly due to lack of mesh resolution - the magnitude of the oscillation indeed diminishes as the grid resolution $N^{2}$ increases. 

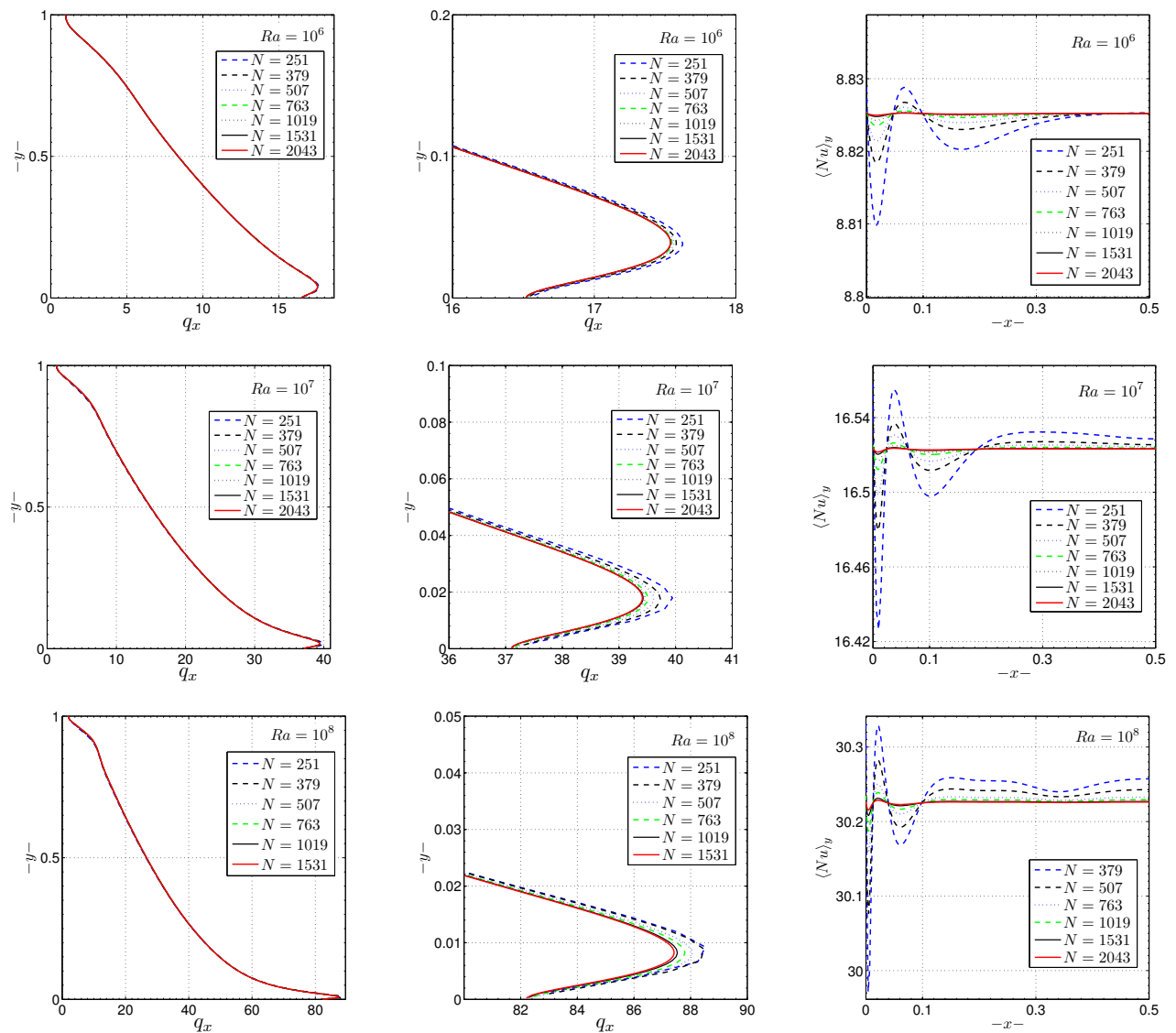

Figure 4: From left to right: the heat flux $q_{x}$ along the hot wall at $x=0$ and an enlarged figure about its maximum near the lower-left corner, and the Nusselt number $\langle\mathrm{Nu}\rangle_{y}(x)$ averaged over $y$ as a function of $x$. From top to bottom: $\mathrm{Ra}=10^{6}, 10^{7}$, and $10^{8}$.

\subsection{Convergence of local hydrodynamic quantities}

Table 4 summarizes the convergence behavior of various local hydrodynamic intensities including the magnitude the stream-function $\psi$ at the cavity center, $\left|\psi_{\text {mid }}\right|$, the maximum magnitude of $\psi,|\psi|_{\max }$ as well as its position, the maximum $x$ and $y$ velocity components, $u_{\max }$ and $v_{\max }$, on the vertical and horizontal symmetric centerline, respectively, as well as their positions. The asymptotic values of these local hydrodynamic intensities are obtained by using the least-square fit with the cubic polynomial of Eq. (51), and will 
be used as the reference solutions to compute the errors. With few exceptions, most measured quantities exhibit a second-order accuracy with the $L_{\infty}$-norm.

\begin{tabular}{|c|c|c|c|c|c|c|c|c|c|}
\hline $\mathrm{Ra}$ & $N^{2}$ & $\left|\psi_{\text {mid }}\right|$ & $|\psi|_{\max }$ & $x$ & $y$ & $u_{\max }$ & $y$ & $v_{\max }$ & $x$ \\
\hline \multirow{9}{*}{$10^{6}$} & $251^{2}$ & 16.4010 & 16.8308 & 0.1490 & 0.5471 & 64.8223 & 0.8497 & 220.5259 & 0.0378 \\
\hline & $379^{2}$ & 16.3965 & 16.8283 & 0.1494 & 0.5472 & 64.8295 & 0.8498 & 220.5370 & 0.0378 \\
\hline & $507^{2}$ & 16.3920 & 16.8207 & 0.1497 & 0.5470 & 64.8341 & 0.8499 & 220.5429 & 0.0378 \\
\hline & $763^{2}$ & 16.3902 & 16.8184 & 0.1499 & 0.5469 & 64.8332 & 0.8499 & 220.5539 & 0.0378 \\
\hline & $1019^{2}$ & 16.3885 & 16.8152 & 0.1500 & 0.5469 & 64.8337 & 0.8499 & 220.5589 & 0.0378 \\
\hline & $1531^{2}$ & 16.3874 & 16.8130 & 0.1501 & 0.5468 & 64.8342 & 0.8499 & 220.5624 & 0.0378 \\
\hline & $2043^{2}$ & 16.3871 & 16.8125 & 0.1502 & 0.5468 & 64.8342 & 0.8499 & 220.5631 & 0.0378 \\
\hline & $\infty$ & 16.3869 & 16.8121 & 0.1502 & 0.5468 & 64.8344 & 0.8499 & 220.5638 & 0.0378 \\
\hline & $n$ & 2.2038 & 2.2245 & 1.9492 & 1.8542 & 2.1090 & 2.0112 & 2.1261 & 2.0008 \\
\hline \multirow{9}{*}{$10^{7}$} & $251^{2}$ & $29.4723 *$ & $30.3375 *$ & 0.0843 & $0.5571 *$ & 148.3414 & 0.8795 & 699.0722 & 0.0214 \\
\hline & $379^{2}$ & $29.4574 *$ & $30.3489 *$ & 0.0848 & $0.5578 *$ & 148.4833 & 0.8794 & 699.1096 & 0.0214 \\
\hline & $507^{2}$ & 29.4153 & 30.2674 & 0.0851 & 0.5569 & 148.5291 & 0.8794 & 699.2071 & 0.0213 \\
\hline & $763^{2}$ & 29.4012 & 30.2469 & 0.0854 & 0.5567 & 148.5610 & 0.8793 & 699.2341 & 0.0213 \\
\hline & $1019^{2}$ & 29.3838 & 30.2104 & 0.0856 & 0.5562 & 148.5718 & 0.8793 & 699.2763 & 0.0213 \\
\hline & $1531^{2}$ & 29.3715 & 30.1846 & 0.0857 & 0.5559 & 148.5795 & 0.8793 & 699.3063 & 0.0213 \\
\hline & $2043^{2}$ & 29.3689 & 30.1798 & 0.0858 & 0.5558 & 148.5821 & 0.8793 & 699.3121 & 0.0213 \\
\hline & $\infty$ & 29.3667 & 30.1760 & 0.0858 & 0.5558 & 148.5852 & 0.8793 & 699.3166 & 0.0213 \\
\hline & $n$ & 2.1845 & 2.2415 & 2.1515 & 2.1638 & 2.0724 & 2.0389 & 2.2358 & 2.1612 \\
\hline \multirow{8}{*}{$10^{8}$} & $379^{2}$ & 52.8567 & 54.8698 & 0.0469 & 0.5594 & 315.0766 & 0.9239 & 2221.4020 & 0.0121 \\
\hline & $507^{2}$ & 52.6243 & 54.4205 & 0.0473 & 0.5564 & 317.7138 & 0.9256 & 2221.8299 & 0.0120 \\
\hline & $763^{2}$ & 52.5458 & $54.3077 *$ & 0.0476 & $0.5560 *$ & 319.8787 & 0.9268 & $2221.9532 *$ & 0.0120 \\
\hline & $1019^{2}$ & 52.4478 & 54.1060 & 0.0478 & 0.5545 & 320.7388 & 0.9273 & 2222.1450 & 0.0120 \\
\hline & $1531^{2}$ & 52.3780 & 53.9625 & 0.0480 & 0.5533 & 321.3676 & 0.9276 & 2222.2806 & 0.0120 \\
\hline & $2043^{2}$ & 52.3634 & 53.9352 & 0.0480 & 0.5532 & 321.5900 & 0.9277 & 2222.3043 & 0.0120 \\
\hline & $\infty$ & 52.3508 & 53.9149 & 0.0481 & 0.5530 & 321.9075 & 0.9278 & 2222.3279 & 0.0119 \\
\hline & $n$ & 2.1639 & 2.2447 & 1.7227 & 2.2573 & 1.8611 & 1.9051 & 2.1692 & 1.8226 \\
\hline
\end{tabular}

Table 4: Convergence behaviors of $\left|\psi_{\text {mid }}\right|,|\psi|_{\max }$ and its position, $u_{\max }$ and $v_{\max }$ as well as their positions. Note: the data that do not fit well with the interpolating polynomial have been excluded from the computation of the asymptotic values and they are marked with asterisk*.

\subsection{Benchmark results}

We now compare our results of the Nusselt numbers and the hydrodynamic quantities with existing benchmark data. The second-order finite difference (FD) solutions of de Vahl Davis [14] for the lower Rayleigh numbers $\mathrm{Ra}=10^{3}, 10^{4}, 10^{5}$, and $10^{6}$ remain a comprehensive benchmark. To our 
knowledge, the Chebyshev pseudo-spectral (PS) solutions of Le Quéré [15] are the most accurate ones for higher Rayleigh numbers $\mathrm{Ra}=10^{7}$ and $10^{8}$. There exist more recent results by using the finite element (FE) method [18], finite difference method $[14,16]$, and the lattice Boltzmann method with coarser meshes [4]. Table 5 compiles the data for the Nusselt numbers and their positions obtained with various methods. The LB results of the present work are the asymptotic values in Tab. 5, obtained by using the Richardson extrapolation. With the exception of two cases $\left(\mathrm{Nu}_{\min }\right.$ at $\mathrm{Ra}=10^{7}$ and $\langle\mathrm{Nu}\rangle$ at $\mathrm{Ra}=10^{8}$ ) which miss the last significant digit, our LB results and the pseudo-spectral benchmark data [15] agree with each other in all significant digits.

\begin{tabular}{c|l|c|l|l|l|l|l}
\hline $\mathrm{Ra}$ & Method & $\langle\mathrm{Nu}\rangle$ & $\mathrm{Nu}_{0}$ & $\mathrm{Nu}_{1 / 2}$ & $\mathrm{Nu}_{\max }$ & \multicolumn{1}{|c}{$y$} & $\mathrm{Nu}_{\min }$ \\
\hline \multirow{7}{*}{$10^{6}$} & Present & 8.8252 & 8.8252 & 8.8252 & 17.5360 & 0.0392 & 0.9795 \\
& PS [15] & - & 8.825 & 8.825 & 17.536 & 0.039 & 0.9795 \\
& FD [14] & 8.800 & 8.817 & 8.799 & 17.925 & 0.0378 & 0.989 \\
& FD [16] & - & 8.8216 & - & 17.5087 & 0.0396 & 0.9787 \\
& LB [4] & 8.8253 & 8.8192 & 8.8254 & 17.5274 & 0.0392 & 0.9769 \\
& FE [18] & - & 8.8811 & - & 17.5308 & 0.0377 & 0.9845 \\
\hline \multirow{5}{*}{$10^{7}$} & Present & 16.5231 & 16.5230 & 16.5231 & 39.3950 & 0.0180 & 1.3659 \\
& PS [15] & - & 16.523 & 16.523 & 39.39 & 0.018 & 1.366 \\
& FD [16] & - & 16.5106 & - & 39.2540 & 0.0179 & 1.3655 \\
& FE [18] & - & 16.3869 & - & 41.0247 & 0.0390 & 1.3799 \\
\hline \multirow{3}{*}{$10^{8}$} & Present & 30.2251 & 30.2241 & 30.2251 & 87.2454 & 0.0083 & 1.9195 \\
& PS [15] & - & 30.225 & 30.225 & 87.24 & 0.008 & 1.919 \\
& FE [18] & - & 29.6256 & - & 91.2095 & 0.0067 & 2.0440 \\
\hline
\end{tabular}

Table 5: Comparison of the Nusselt numbers and their locations with existing benchmark data.

Table 6 compiles the data for the measured hydrodynamic intensities and their positions obtained with various methods. It should also be noted that in Le Quéré's work [15], the velocity is normalized by $\sqrt{\mathrm{Ra}} \kappa / L$, as opposed to $\kappa / L$. Thus the values of the stream-function $\psi$ and the velocity $\boldsymbol{u}$ in Le Quéré's work [15] given in Tab. 6 have been multiplied by $\sqrt{\mathrm{Ra}}$. For the case of $\mathrm{Ra}=10^{6}$, the present LB results and the PS data [15] are essentially identical. And for the cases of $\mathrm{Ra}=10^{7}$ and $10^{8}$, the present LB results and the PS data [15] agree with each other with at least three significant digits 
except for the case of $\left|\psi_{\max }\right|$ at $\mathrm{Ra}=10^{8}$, for which the LB result differs from the PS data for about $0.12 \%$.

\begin{tabular}{c|l|l|l|l|l|l|l|l|l}
\hline Ra & Method & $\left|\psi_{\text {mid }}\right|$ & $|\psi|_{\max }$ & $x$ & $y$ & $u_{\max }$ & \multicolumn{1}{|c|}{$y$} & \multicolumn{1}{c}{$v_{\max }$} & \multicolumn{1}{c}{$x$} \\
\hline \multirow{6}{*}{$10^{6}$} & Present & 16.3868 & 16.8118 & 0.1502 & 0.5468 & 64.8344 & 0.8499 & 220.5644 & 0.0378 \\
& PS [15] & 16.386 & 16.811 & 0.150 & 0.547 & 64.83 & 0.850 & 220.6 & 0.038 \\
& FD [14] & 16.32 & 16.750 & 0.151 & 0.547 & 64.63 & 0.850 & 219.36 & 0.0379 \\
& FE [17] & 16.3860 & 16.8110 & 0.1504 & 0.5470 & 64.8340 & 0.8499 & 220.5650 & 0.0378 \\
& LB [4] & 16.3868 & 16.8149 & 0.1503 & 0.5468 & 64.8336 & 0.8499 & 220.5658 & 0.0378 \\
& FD [16] & 16.3863 & 16.8107 & - & - & 64.8308 & 0.8501 & 220.5675 & 0.0377 \\
& FE [18] & - & - & - & - & 64.6912 & 0.8460 & 220.8331 & 0.0380 \\
\hline \multirow{5}{*}{$10^{7}$} & Present & 29.3653 & 30.1760 & 0.0858 & 0.5558 & 148.5852 & 0.8793 & 699.3224 & 0.0213 \\
& PS [15] & 29.3617 & 30.1649 & 0.086 & 0.556 & 148.5954 & 0.879 & 699.1795 & 0.021 \\
& FD [16] & 29.3562 & 30.1553 & - & - & 148.5695 & 0.8794 & 699.2991 & 0.0213 \\
& FE [18] & - & - & - & - & 145.2666 & 0.8845 & 703.2526 & 0.0215 \\
\hline \multirow{3}{*}{$10^{8}$} & Present & 52.3508 & 53.9149 & 0.0481 & 0.5530 & 321.9063 & 0.9279 & 2222.3279 & 0.0120 \\
& PS [15] & 52.32 & 53.85 & 0.048 & 0.553 & 321.9 & 0.928 & 2222. & 0.012 \\
& FE [18] & - & - & - & - & 283.0689 & 0.9455 & 2223.4424 & 0.0130 \\
\hline
\end{tabular}

Table 6: Comparison of the measured hydrodynamic intensities with existing benchmark data.

\section{Conclusions}

In this work we use the multiple-relaxation-time lattice Boltzmann model with dual distributions [4] to simulate thermal flows in the two-dimensional square cavity with differentially heated vertical walls. The MRT-TLBE uses the D2Q9 model and D2Q5 model for mass-momentum and temperature conservations, respectively. The D2Q9+D2Q5 MRT-LB model essentially solves the thermal fluid system with the Boussinesq approximation. The flow parameters are the Prandtl number $\operatorname{Pr}=0.71$ (for air) and the Rayleigh number $\mathrm{Ra}=10^{6}, 10^{7}$, and $10^{8}$. The Mach number is fixed to be $\mathrm{Ma}=0.1$ in our simulations. With the given Rayleigh numbers, the flow is steady.

We now summarize our observations in this work as the following. First, we emphasize that the MRT collision model is imperative to achieve accurate boundary conditions and numerical stability. We would also like to point out that the LBGK model fails in both these aspects. Second, with the given flow conditions, we have shown that the MRT-TLBE model is 
second-order accurate for flow fields with $L_{2}$-norm, global quantities, such as Nusselt numbers, and local hydrodynamic intensities as well as their positions, with $L_{\infty}$-norm. Third, our results demonstrate that the MRT-LB model can reproduce numerical results of benchmark quality for internal incompressible thermal flows. Our results agree well with existing benchmark data obtained by using traditional methods. Finally, the LB algorithm has been implemented on GPU, and can achieve a speed of 560 million millions site-updates per second with double precision (1080 millions site-updates per second with single precision), which allows us to complete the simulations on a mesh as large as $2043^{2}$ within 15 hours.

\section{Acknowledgments}

LSL would like to would like to acknowledge the support of the Richard F. Barry Jr. Endowment at the Old Dominion University (ODU) and a visiting fellowship from the Isaac Newton Institute of Mathematical Sciences, University of Cambridge, UK, for the period of July 6 - August 3, 2013, during which part of this work was carried out.

\section{References}

[1] G. McNamara, A. Garcia, B. Alder, A hydrodynamically correct thermal lattice Boltzmann model, J. Stat. Phys. 87 (5/6) (1997) 1111-1121.

[2] P. Lallemand, L.-S. Luo, Theory of the lattice Boltzmann method: Acoustic and thermal properties in two and three dimensions, Phys. Rev. E 68 (3) (2003) 036706.

[3] J. Eggels, J. Somers, Numerical-simulation of free convective flow using the lattice-Boltzmann scheme, Int. J. Heat Fluid Flow 16 (5) (1995) $357-364$.

[4] J. Wang, D. Wang, P. Lallemand, L.-S. Luo, Lattice Boltzmann simulations of thermal convective flows in two dimensions, Comput. Math. Appl. 65 (2) (2013) 262-286. doi:\{10.1016/j.camwa.2012.07.001\}. 
[5] P. Lallemand, L.-S. Luo, Hybrid finite-difference thermal lattice Boltzmann equation, Int. J. Mod. Phys. B 17 (1/2) (2003) 41-47.

[6] D. d'Humières, Generalized lattice-Boltzmann equations, in: B. D. Shizgal, D. P. Weave (Eds.), Rarefied Gas Dynamics: Theory and Simulations, Vol. 159 of Prog. Astronaut. Aeronaut., AIAA, Washington, D.C., 1992, pp. 450-458.

[7] P. Lallemand, L.-S. Luo, Theory of the lattice Boltzmann method: Dispersion, dissipation, isotropy, Galilean invariance, and stability, Phys. Rev. E 61 (6) (2000) 6546-6562.

[8] I. Ginzburg, Equilibrium-type and link-type lattice Boltzmann models for generic advection and anisotropic-dispersion equation, Adv. Water Res. 28 (11) (2005) 1171-1195.

[9] I. Ginzburg, Generic boundary conditions for lattice Boltzmann models and their application to advection and anisotropic dispersion equations, Adv. Water Res. 28 (11) (2005) 1196-1216.

[10] I. Ginzburg, Variably saturated flow described with the anisotropic lattice Boltzmann methods, Comput. Fluids 35 (8-9) (2006) 831-848.

[11] I. Ginzburg, D. d'Humières, Lattice Boltzmann and analytical modeling of flow processes in anisotropic and heterogeneous stratified aquifers, Adv. Water Res. 30 (11) (2007) 2202-2234.

[12] I. Ginzburg, Lattice Boltzmann modeling with discontinuous collision components: Hydrodynamic and advection-diffusion equations, J. Stat. Phys. 126 (1) (2007) 157-206.

[13] G. de Vahl Davis, I. P. Jones, Natural-convection in a square cavity: A comparison exercise, Int. J. Numer. Meth. Fluids 3 (3) (1983) 227-248. 
[14] G. de Vahl Davis, Natural-convection of air in a square cavity: A benchmark numerical-solution, Int. J. Numer. Meth. Fluids 3 (3) (1983) 249 264.

[15] P. Le Quéré, Accurate solutions to the square thermally driven cavity at high Rayleigh number, Comput. Fluids 20 (1) (1991) 29-41.

[16] Z. Tian, Y. Ge, A fourth-order compact finite difference scheme for the steady stream function-vorticity formulation of the NavierStokes/Boussinesq equations, Int. J. Numer. Meth. Fluids 41 (5) (2003) 495-518. doi:10.1002/fld. 444.

[17] J. T. Holdeman, J. W. Kim, Computation of incompressible thermal flows using Hermite finite elements, Comput. Methods Appl. Mech. Engrg. 199 (49-52) (2010) 3297-3304. doi:\{10.1016/j.cma.2010.06. 036\}.

[18] D. Mayne, A. S. Usmani, M. Crapper, $h$-Adaptive finite element solution of high Rayleigh number thermally driven cavity problem, Int. J. Numer. Meth. Heat Fluid Flow 10 (5-6) (2000) 598-615.

[19] M. Hortmann, M. Perić, G. Scheuerer, Finite volume multigrid prediction of laminar natural-convection: Bench-mark solutions, Int. J. Numer. Meth. Fluids 11 (2) (1990) 189-207.

[20] R. Henkes, C. Hoogendoorn, Scaling of the laminar natural-convection flow in a heated square cavity, Int. J. Heat Mass Trans. 36 (11) (1993) 2913-2925. doi:10.1016/0017-9310 (93)90110-R.

[21] N. Mai-Duy, T. Tran-Cong, A control volume technique based on integrated RBFNs for the convection-diffusion equation, Numer. Methods Part. Diff. Eq. 26 (2) (2010) 426-447. doi:10.1002/num. 20444.

[22] D. d'Humières, M. Bouzidi, P. Lallemand, Thirteen-velocity threedimensional lattice Boltzmann model, Phys. Rev. E 63 (2001) 066702. 
[23] D. d'Humières, I. Ginzburg, M. Krafczyk, P. Lallemand, L.-S. Luo, Multiple-relaxation-time lattice Boltzmann models in three-dimensions, Philos. Trans. R. Soc., A 360 (1792) (2002) 437-451.

[24] I. Ginzburg, F. Verhaeghe, D. d'Humières, Two-relaxation-time lattice Boltzmann scheme: About parametrization, velocity, pressure and mixed boundary conditions, Commun. Comput. Phys. 3 (2008) 427-478.

[25] I. Ginzburg, F. Verhaeghe, D. d'Humières, Study of simple hydrodynamic solutions with the two-relaxation-times lattice Boltzmann scheme, Commun. Comput. Phys. 3 (3) (2008) 519-581.

[26] I. Ginzburg, D. d'Humières, Multireflection boundary conditions for lattice Boltzmann models, Phys. Rev. E 68 (6) (2003) 066614.

[27] C. Pan, L.-S. Luo, C. Miller, An evaluation of lattice Boltzmann schemes for porous medium flow simulation, Comput. Fluids 35 (8/9) (2006) 898909 .

[28] L.-S. Luo, W. Liao, X. Chen, Y. Peng, W. Zhang, Numerics of the lattice Boltzmann method: Effects of collision models on the lattice Boltzmann simulations, Phys. Rev. E 83 (5) (2011) 056710.

[29] X. He, L.-S. Luo, Lattice Boltzmann model for the incompressible Navier-Stokes equation, J. Stat. Phys. 88 (3/4) (1997) 927-944.

[30] F. Dubois, P. Lallemand, Towards higher order lattice Boltzmann schemes, J. Stat. Mech. (2009) P06006.

[31] Y. H. Qian, Y. Zhou, Complete Galilean-invariant lattice BGK models for the Navier-Stokes equation, Europhys. Lett. 42 (4) (1998) 359-364. doi:10.1209/epl/i1998-00255-3.

[32] I. Ginzburg, Truncation errors, exact and heuristic stability analysis of two-relaxation-times lattice Boltzmann schemes for anisotropic 
advection-diffusion equation, Commun. Comput. Phys. 11 (5) (2012) 1439-1502.

[33] P. J. Dellar, An interpretation and derivation of the lattice Boltzmann method using Strang splitting, Comput. Math. Appl. 65 (2013) 129-141.

[34] Z. Guo, C. Zheng, B. Shi, Discrete lattice effects on the forcing term in the lattice Boltzmann method, Phys. Rev. E 65 (2002) 046308. 